\title{
Putting Stiffness where it's needed: Optimizing the Mechanical Response of Multi-Material Structures
}

\author{
Arouna Patouossa Mounchili ${ }^{1}$, Stefan Bosse ${ }^{2}$, Dirk Lehmhus ${ }^{1}$, and Adrian Struss ${ }^{1}$ \\ ${ }^{1}$ Fraunhofer Institute for Manufacturing Technology and Advanced Materials IFAM, Dept. of Casting \\ Technology and Lightweight Construction, 28359 Bremen, Germany \\ ${ }^{2}$ University of Bremen, Department of Computer Science, 28359 Bremen, Germany
}

\begin{abstract}
Manufacturing processes are increasingly adapted to multimaterial part production to facilitate lightweight design via improvement of structural performance. The difficulty lies in determining the optimum spatial distribution of the individual materials. Multi-Phase Topology Optimization (MPTO) achieves this aim via iterative, linear-elastic Finite Element (FE) simulations providing element- and part-level strain energy data under a given design load and using it to redistribute predefined material fractions to minimize total strain energy. The result us a part configuration offering maximum stiffness. The present study implements different material redistribution and optimization techniques and compares them in terms of optimization results and performance: Genetic algorithms are matched against simulated annealing, the latter with and without physicsbased constraints. Both types employ partial randomization in generating new configurations to avoid settling into local rather than global minima of the objective function. This allows exploring a larger fraction of the full search space than accessed by classic gradient-based algorithms. Evaluation of the objective function depends on FE simulation, a computationally intensive task. Minimizing the required number of simulation runs is the task of the aforementioned constraints. The methodology is validated via a three point bending test scenario.
\end{abstract}

\section{Introduction}

\subsection{Multi-material manufacturing: Techniques and motivations}

Recent developments in manufacturing engineering have opened up several new paths towards realizing multi-material components. Prominent among these are additive manufacturing (AM) techniques, many of which are directly suited to select local material compositions, while others can be adapted to at least modify local compositions and thus properties [1-3]. In general, examples of either kind exist for arbitrary matrix material classes (polymers [4], metals [5], ceramics [6]). Beyond AM, more conventional manufacturing techniques like metal casting can be employed to generate multi-material structures via approaches like compound or hybrid casting [7,8], the latter transgressing the boundaries of 
material classes by combining metals and plastics by reversing the processing sequence already known from overmolding [9]. The motivation behind realizing multi-material structures may either be the improvement of functional or structural properties, or both. Of interest for the present study are cases where the 3D material distribution is not periodic and depends on the components' design loads, where loads may signify mechanical, thermal or electrical parameters. State of the art examples of this kind include steel-copper, heat spreading die inserts for high pressure die casting molds produced via directed energy deposition AM techniques. In contrast, enhancing mechanical properties - notably stiffness - within a given design space was pursued in a study by Schittenhelm et al., using compound casting to embed a steel reinforcement within an aluminum casting [7]. The optimum geometry of the reinforcing steel part was defined using the fundamental MPTO approach as originally described by Burblies and Busse [10].

\subsection{Multi-Phase Topology Optimization (MPTO)}

The MPTO approach was published in 2006 by Burblies and Busse [10]. It represents a methodology to distribute several fixed material fractions over a part volume in such a way that stiffness is maximized under a given load. Its fundamental principle uses the fact that under linear-elastic conditions, maximum stiffness coincides with a minimum of the total strain energy of the loaded part. This observation also defines the objective function to be minimized during optimization. To do so, Burblies and Busse followed a simulated annealing approach enhanced by a physics-based constraint: First, an FE model of the problem is created covering part geometry, boundary conditions and loads. Material properties are then associated to the individual elements: Number of different materials, defined by their elastic properties (Young's modulus, Poisson's ratio) and volume fraction are defined the start and maintained throughout the optimization. Secondly, material associations are arbitrarily distributed over the FE model. A first FE simulation provides each element's and the entire part's strain energy. For each element, the product of element volume and element-based strain energy is calculated, and the elements are sorted in descending order based on this product's value. Following this, materials properties are reshuffled: Highest stiffness materials are associated with elements at the top of the list, introducing the aforementioned physics-based constraint. The result is a new configuration of the FE model in terms of the spatial distribution of materials. In order to avoid getting trapped in a local minimum of the objective function, a random element is added at this stage by arbitrarily exchanging a limited number of finite element-material associations. The updated configuration is then subjected to another FE simulation, after which the above steps are repeated.

The innovative aspect of this study lies in the comparison of alternative optimization algorithms applied to the multi-phase topology optimization problem. While the original constrained simulated annealing approach chosen by Burblies and Busse [10] is included as reference, both an entirely random and an evolutionary approach are contrasted to it in terms of optimization results achieved and required computational effort.

\section{Implementation}

Three different types of algorithms were compared in the present study, two of them based on a simulated annealing approach, the third applying a genetic algorithm. Common to these is a distinction between inner and outer steps: As inner steps, we define any attempt of the algorithm to identify an improved configuration, irrespective of the success of this attempt. As outer steps, we define those steps in the course of which the current optimum is changed. 


\subsection{Simulated Annealing}

\subsubsection{Unconstrained Algorithm}

As a starting point of the investigation, a simplistic, unconstrained simulated annealing algorithm [13] is used that has been implemented with Monte Carlo simulation for material distribution. New configurations of the problem are created in a random manner, without any underlying principle that would govern the association of material properties. There is a linear material distribution vector $\vec{D}$ that maps a discrete set of material properties $\{m 1, m 2, .$.$\} on$ elements (with fractions $n \mathrm{i}$ ) of a three-dimensional structure for optimization. The material properties are initially randomly distributed using shuffling, shown in Alg. 1, line 2. The SA algorithm consists of two nested loops. The inner loop searches for a better solution (inner step), and the outer loop anneals the optimization by decreasing the temperature and accepting the current solution for the next iteration (outer step). There is a small probability that a worse solution $\left(E^{\prime}>E\right)$ is accepted which is deliberately implemented to allow the algorithm to escape from local minima of the observable. This faculty is provided by the first expression in line 5 . Each time a new configuration was created by randomly swapping a portion $\eta$ of the material properties (line 7), the strain energy is computed via an FEM simulation. Material properties are associated to volume elements on element level.

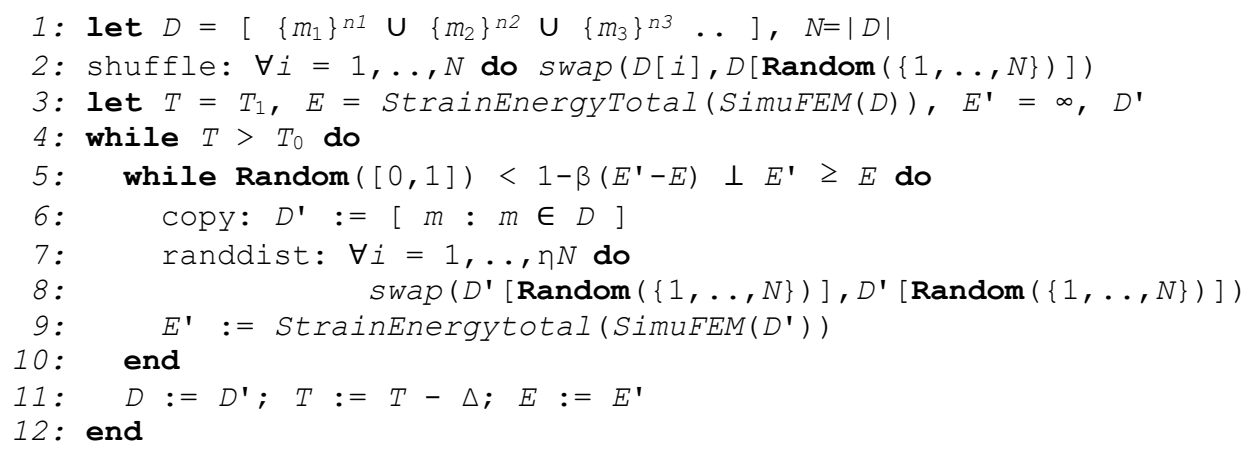

Alg. 1. Unconstrained optimization starting with a randomized distribution using shuffling (FisherYates alg.). $\overrightarrow{\mathrm{D}}$ is a linear vector representing the structure elements material property assignment (material properties $m_{i}$ with fraction $n_{i} / N,|\vec{D}|=N, N=\Sigma n_{i}$ ). E and $E^{\prime}$ are computed total strain energy values of entire structure. [..] are ordered and $\{.$.$\} unordered lists.$

Three different levels of material property change were compared. These are described by the fraction of - arbitrarily chosen - elements affected by such change during each inner step: The actual values chosen were $\eta=1,5$ and $25 \%$.

\subsubsection{Physics-based Constraint}

The original realization of the MPTO approach already uses a physically motivated constraint to achieve faster convergence of the simulation runs. The fundamental idea is that finite elements which exhibit high levels of strain energy while having low stiffness material properties should be associated with high elastic moduli instead. The result would be an overall reduction of strain energy, and thus the increase in stiffness aspired to [10]. The basic approach is shown in Alg. 2. In extension to Alg. 1 and random material property swapping, material properties are sorted based on the strain energy of the respective element. 
As in the case of unconstrained simulated annealing, different levels of material exchange per inner step were compared. These include $\eta=1,5$ and $25 \%$, plus $0 \%$, the latter implying that only the chosen constraint is active in redistributing materials, and no stochastic element modification is involved in this process.

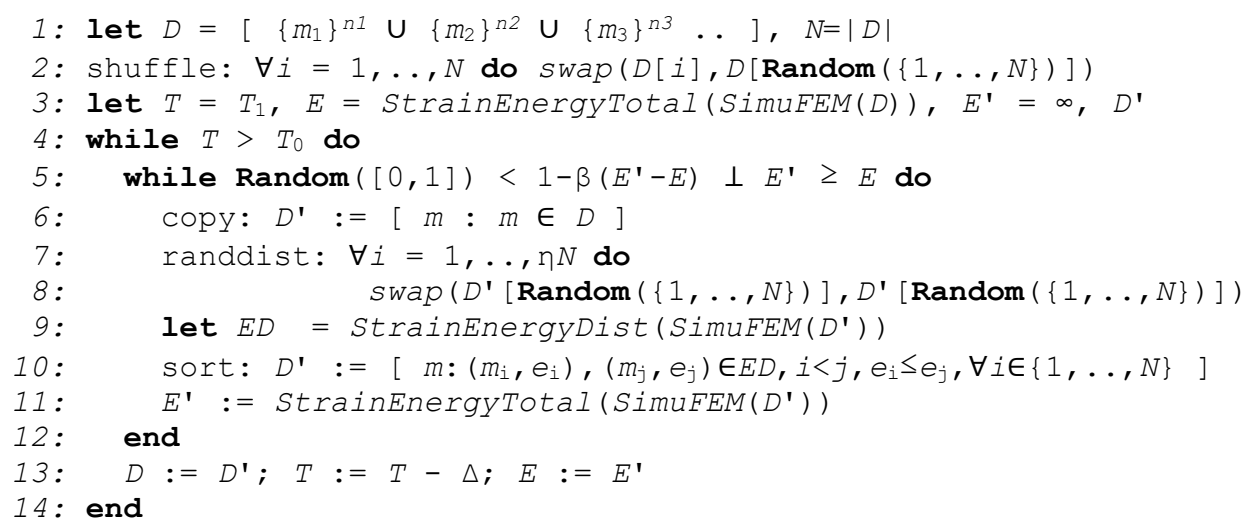

Alg. 2. Constrained optimization starting with a randomized distribution and applying sorting of material properties of element. The associated vector ED consists of $(\mathrm{m}, \mathrm{e})$ tuples, $\mathrm{e}$ is the element strain energy, finally sorted by energy relation and mapped again to a material property vector.

\title{
2.2 Genetic Algorithm
}

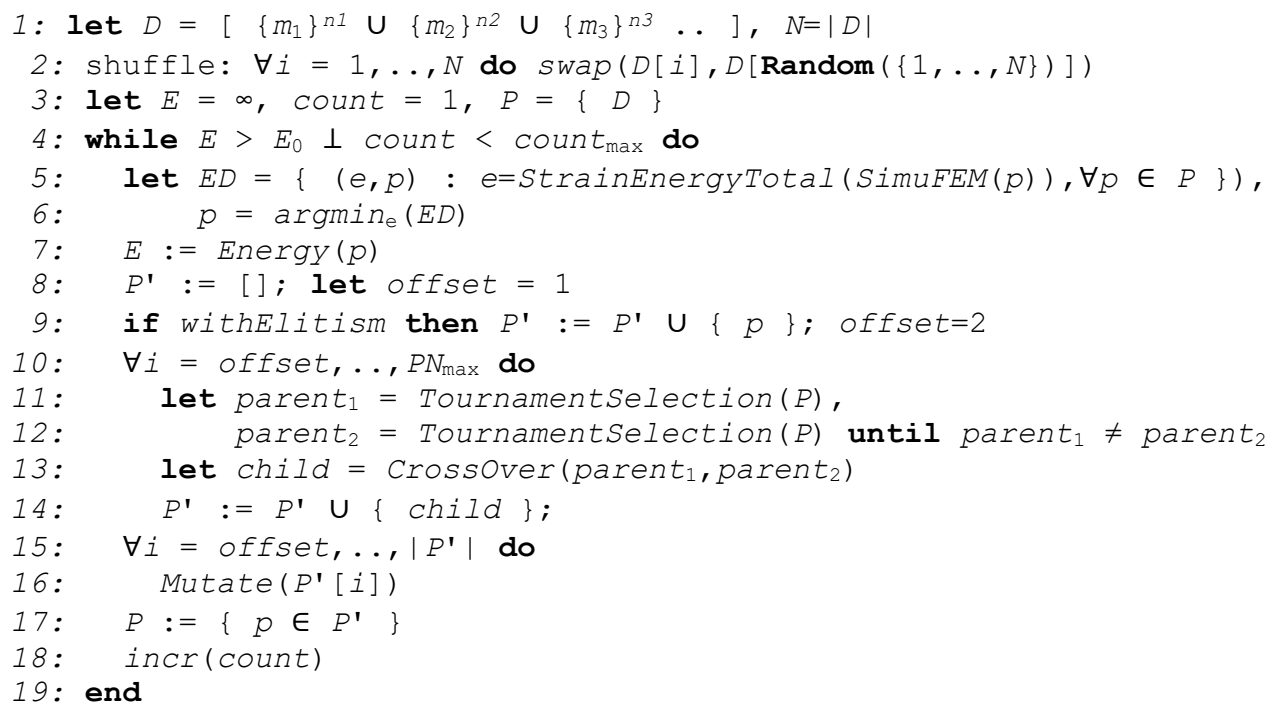

\begin{abstract}
Alg. 3. Genetic Algorithm. A population $\mathrm{P}$ consists of multiple material property solutions (chromosomes) that are used to find the best solution with the lowest total strain energy and to construct new solutions from the previous population set. The maximal population size is limited by $\mathrm{PN}_{\max }$.
\end{abstract}

The genetic algorithm [11,12] is similar to simulated annealing in using randomized generation of new solutions of the material distribution, too. But in contrast to simulated annealing, the this algorithm constructs a set of solutions from randomized shuffling 
(mutation) and partial combination (crossover) based on suitable, selected parent solutions, as shown below in Alg. 3. A fitness function measures the total strain energy, and the best solutions are selected based on this measure.

\subsection{Test Scenario}

In order to evaluate the various algorithms, a common test scenario was defined. Complexity of this scenario, which served as a starting point for comparison of algorithms and was meant to yield initial results fast, was intentionally chosen to be low.

The scenario represents a three point bending test with asymmetric load introduction. The sample itself is of cuboid shape with dimensions of $10 \mathrm{~mm} \times 10 \mathrm{~mm} \times 150 \mathrm{~mm}$. Two point loads of $200 \mathrm{~N}$ each are introduced at opposite upper lateral edges of the beam at a longitudinal position equivalent to $1 / 3$ of the sample's length from one end. The ends of the beam also mark the position of the vertical supports. The beam itself consists of equal volume fractions of three different materials, namely "aluminum" (Young's modulus $70 \mathrm{GPa}$ ), "copper" (110 GPa) and "steel" (200 GPa). Poisson's ratio was set to 0.3 in all cases. Since only linear elastic simulations were performed, no further material properties were required. The number of elements is 459 , all of equal size, with 832 nodes.

\section{Results and Discussion}

Fig. 1 below depicts the outcome of four separate runs of the unconstrained simulated annealing algorithm. As can be seen, the improvement in strain energy achieved over 1000 inner steps, i. e. 1000 times randomly reshuffling the configuration, falls below $10 \%$.
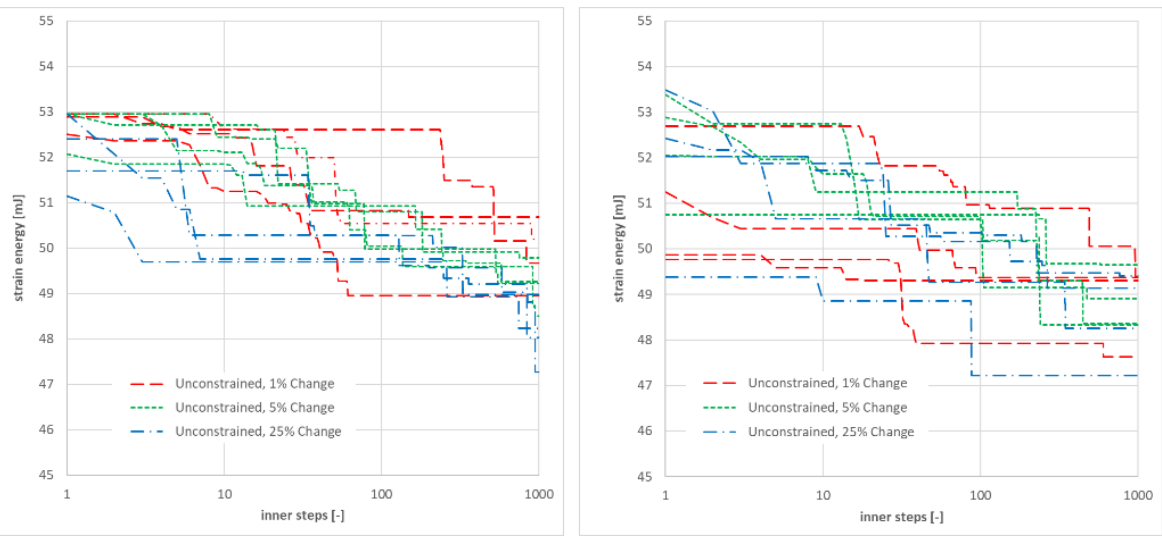

Fig. 1. Development of strain energy over inner simulation steps for unconstrained simulated annealing (level of change 1, 5 and $25 \%$ ). Left, 4 runs based on the same initial configuration (associated strain energy $52.946 \mathrm{~mJ}$ ), right, 4 runs based on different initial configurations.

In contrast to Fig. 1, Fig. 2 shows the influence of the physical constraint introduced with the constrained simulated annealing algorithm. Most noteworthy is the fact that in this case, already after the very first step, strain energy levels between 32.8 and $33.2 \mathrm{~mJ}$ are reached in all cases, by far exceeding the achievements of the unconstrained algorithm. Closer observation shows that, as expected, the constrained algorithm without random elements $(0 \%$ level of element property exchange) yields identical results if the initial configuration is the same (see Fig. 2, left hand side). Introduction of a certain proportion of random exchange can lead to a further, though slight, reduction of strain energy in the present example. However, the smaller the chosen level of the random element is, the higher is the 
improvement - this is obvious both in cases starting from the same and from different initial configurations (see Fig. 2, right).
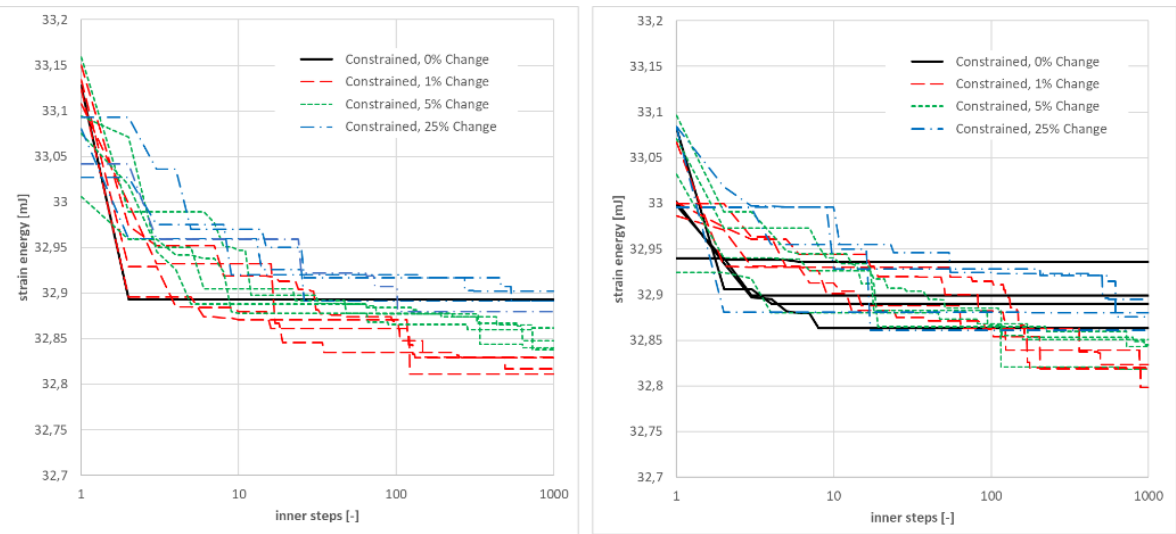

Fig. 2. Development of strain energy over outer simulation steps for constrained simulated annealing (level of change $\mathbf{0}, \mathbf{1}, \mathbf{5}$ and $25 \%$ ). Left, 4 runs based on the same initial configuration (associated strain energy value $52.946 \mathrm{~mJ}$ ), right, 4 runs based on different initial configurations.

Compared to simulated annealing, the genetic algorithm exhibits almost constant progress in each inner step and appears to converge towards an optimum (see Fig. 3). The final levels of strain energy reached after 1000 inner steps are slightly higher than those associated with constrained simulated annealing, but clearly in the same order of magnitude.
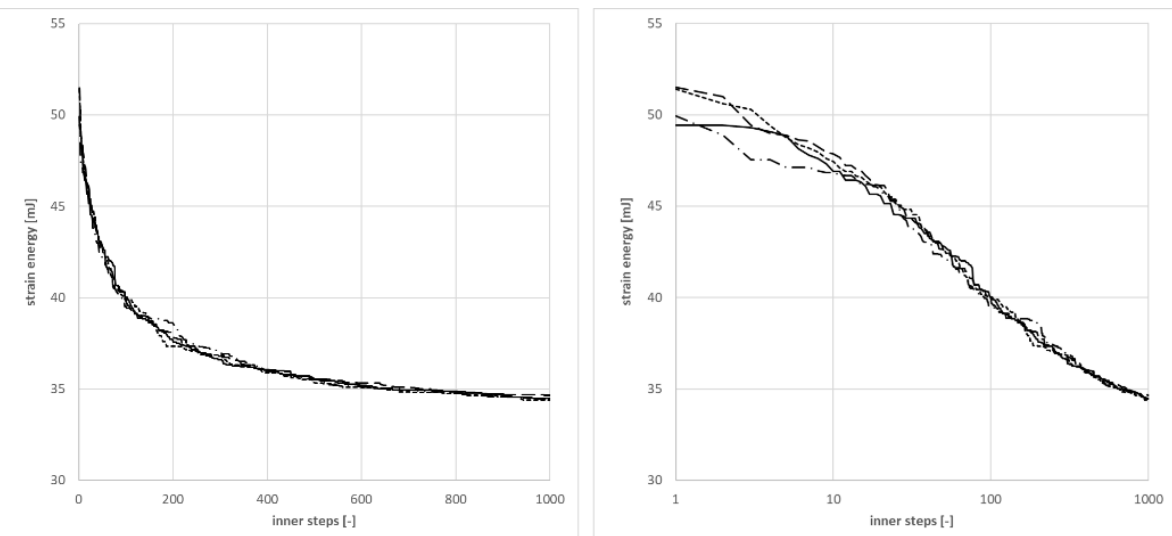

Fig. 3. Development of strain energy over outer simulation steps for the genetic algorithm. Left, linear, right, logarithmic scaling of the coordinate system's horizontal axis.

This said, it must be noted that the genetic algorithm is computationally demanding, as each inner step requires an FEM calculation for a population of configurations rather than for a single one. While an inner step in simulated annealing thus affords either one (unconstrained) or two (constrained) FE simulation runs, the genetic algorithm affords twenty. Fig. 4 visually confirms findings based on the evolution of strain energy values over inner steps. While the unconstrained algorithm shows very limited development, constrained simulated annealing already comes up with an almost perfect configuration after the first step, which is then modified only gradually. In contrast, the genetic algorithm produces a constant evolution towards a configuration which increasingly resembles the output of the constrained simulated annealing algorithm. 


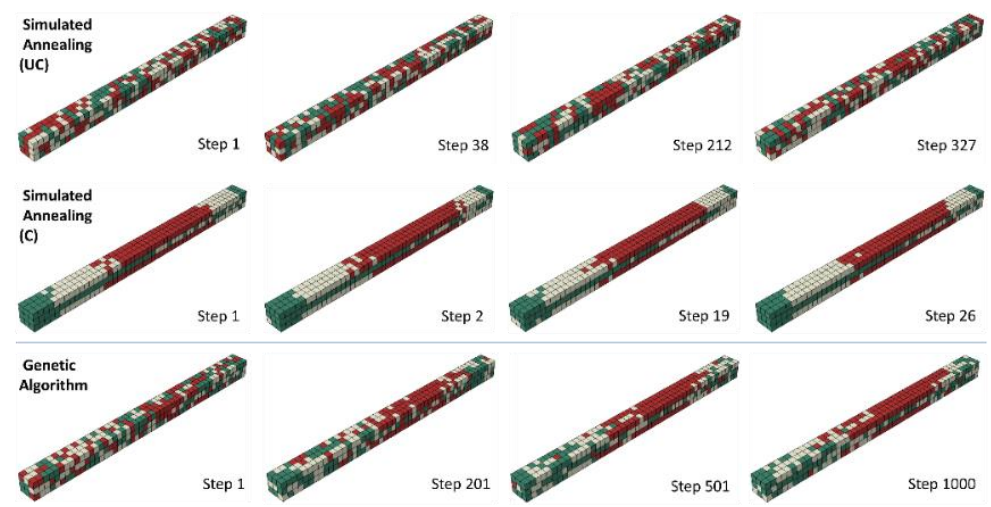

Fig. 4. Comparison of configuration change for increasing numbers of outer steps. Top, unconstrained simulated annealing at $25 \%$ level of change, centre, constrained simulated annealing at $25 \%$ change, bottom, genetic algorithm.

In order to better understand the performance of the unconstrained simulated annealing approach, a random sample of 1000 configurations has been created and the statistical distribution of energies analysed. Fig. 5 displays a histogram of the sample's distribution. To verify whether this observation may be described by a normal distribution, a Shapiro-Wilk test has been executed using the OriginPro software package (Version 2021. OriginLab Corporation, Northampton, MA, USA.). The test statistic obtained was 0,99765, which supports the hypothesis. Average value and standard deviation, 52,78757 and 1,37036 mJ, respectively, can serve as estimators for mean and standard deviation of the basic population. The resulting probability density function is included in Fig. 5.

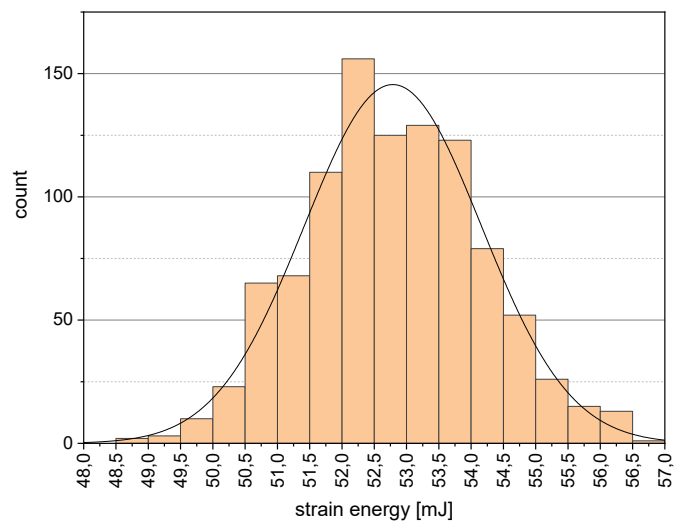

Fig. 5. Histogram of strain energies and associated probability density function as derived from a sample of 1000 random configurations.

Based on the general characteristics of a normal distribution, the likelihood of a random configuration having a strain energy below $48,676 \mathrm{~mJ}$ is roughly $0,135 \%$. It is thus not surprising that after 1000 inner steps, the outcome of the unconstrained simulated annealing algorithm in terms of strain energy largely falls into this order of magnitude. Similarly, the observation may explain qualitatively why increasing levels of change adversely affect the very convincing results obtained via the constrained simulated annealing algorithm when compared to the purely physics-based algorithm without any random exchange of material attributions. At the same time, the fact that a superimposed limited randomization of up to 
$5 \%$ may still lead to improvement underlines that even in this very simple scenario, the purely deterministic approach does not necessarily identify the optimum solution. For problems of greater complexity, the introduction of such stochastic effects is thus highly recommended.

Directly comparing these results with the genetic algorithm's outcome is complicated by the fact that the latter employs a significantly increased number of simulations per step. Nevertheless, it is obvious that it eventually converges both towards strain energy values and configurations matching those obtained via the constrained simulated annealing algorithm. This tendency is clearly visible in Fig. 3 and 4, which at closer scrutiny also show that already at 50 steps - corresponding to 1000 FEM simulations and thus the full number of such runs executed for the unconstrained simulated annealing approach -, a strain energy level below $43 \mathrm{~mJ}$ is reproducibly achieved, clearly outmatching all fully randomized approaches.

\section{Summary and Conclusion}

The comparison of simulated annealing and genetic algorithms has met initial expectations in the sense that unconstrained simulated annealing could be shown to yield no improvements other than those encountered coincidentally based on the statistical distribution of energies in the chosen model system. To confirm this observation, the stochastic distribution of strain energies in random samples has been analysed. The result as such, however, is not a weakness of the study but the necessary establishment of a baseline for further observations. Constraining the simulated annealing approach yields the expected vast improvement both over the unconstrained simulated annealing and the genetic algorithm. Here, for the given test scenario, the number of steps needed to reach what is among the lowest strain energy levels observed in this study is two orders of magnitude below that required by the two other algorithms. However, further improvement is achieved if a stochastic element is maintained. Unlike unconstrained simulated annealing, the genetic algorithm gradually converges to strain energies similar to those associated with constrained simulated annealing, providing an excellent starting point for future studies. These will investigate ways of enhancing the genetic algorithm by introducing physics-based constraints as well as checks to eliminate potentially unproductive simulation runs. Furthermore, the scaling characteristics of the algorithms will be investigated, both in terms of the number of elements and materials.

\section{References}

1. A. Bandyopadhyay, B. Heer. Additive manufacturing of multi-material structures. Mat. Sci. Eng. R: Reports 129, 1-16 (2018)

2. D. Lehmhus, M. Busse, A. von Hehl, E. A. Jägle, State of the art and emerging trends in additive manufacturing: from multi-material processes to $3 \mathrm{D}$ printed electronics. MATEC Web of Conferences 188, 03013 (2018)

3. M. Rafiee, R. D. Farahani, D. Therriault, Multi-material 3D and 4D printing: a survey. Advanced Science 7, 1902307 (2020)

4. Y. Zheng, W. Zhang, D. M. Baca Lopez, R. Ahmad, Scientometric Analysis and Systematic Review of Multi-Material Additive Manufacturing of Polymers. Polymers 13, 1957 (2021)

5. N. E. Putra, M. J. Mirzaali, I. Apachitei, J. Zhou, A. A. Zadpoor, Multi-material additive manufacturing technologies for $\mathrm{Ti}$-, $\mathrm{Mg}$-, and $\mathrm{Fe}$-based biomaterials for bone substitution. Acta Biomaterialia 109, 1-20 (2020)

6. S. Weingarten, R. Johne, U. Scheithauer, T. Moritz, J. Abel, Multi-material CeramicBased Components - Additive Manufacturing of Black-and-white Zirconia 
Components by Thermoplastic 3D-Printing (CerAM - T3DP). J. Vis. Exp. 143, e57538 (2019)

7. D. Schittenhelm, A. Burblies, M. Busse, Stahlverstärkter Aluminiumguss Bauraumreduzierung durch lastfallgerechte Auslegung eines VerbundHecklängsträgers mittels Mehrphasen-Topologieoptimierung. Forschung im Ingenieurwesen 82, 131-147 (2018)

8. D. Lehmhus, A. von Hehl, J. Hausmann, K. Kayvantash, R. Alderliesten, J. Hohe, New materials and processes for transport applications: going hybrid and beyond. Adv. Eng. Mat. 21, 1900056 (2019)

9. A. Hürkamp, A. Dér, S. Gellrich, T. Ossowski, R. Lorenz, D.-A. Behrens, C. Herrmann, K. Dröder, S. Thiede, Integrated computational product and production engineering for multi-material lightweight structures. Int. J. Adv. Manufacturing Technology 110, 2551-2571 (2020)

10. A. Burblies, M. Busse, Computer Based Porosity Design by Multi Phase Topology Optimization, in Proceedings of the Multiscale \& Functionally Graded Materials Conference, FGM, 15-18 October 2006, Honolulu (2006)

11. D. Whitley, A. M. Sutton, Genetic algorithms - a survey of models and methods, In Handbook of natural computing (Springer, Berlin Heidelberg, 2012), 637-671

12. W. Paszkowicz, Genetic algorithms, a nature-inspired tool: survey of applications in materials science and related fields. Materials and Manufacturing Processes 24, 174197 (2009)

13. D. Bertsimas, J. Tsitsiklis, Simulated annealing. Statistical Science 8, 10-15 (1993) 